\title{
Immunogenicity Specimen Assessments Baseline Flag
}

National Cancer Institute

\section{Source}

National Cancer Institute. Immunogenicity Specimen Assessments Baseline Flag. NCI

Thesaurus. Code C117569.

An indication or description that immunogenicity specimen assessment data is a baseline value. 